\title{
Demonstrating TTC-PON Robustness and Flexibility
}

\author{
Eduardo Brandao de Souza Mendes ${ }^{* \dagger}$ \\ CERN \\ E-mail: eduardo.brandao.de.souza.mendes@cern.ch
}

\section{Sophie Baron}

CERN

E-mail: sophie.baron@cern.ch

\section{Csaba Soos}

CERN

E-mail: csaba.soos@cern.ch

\section{Logan Saint-Germain}

Universite de Bordeaux II

E-mail: logansaintgermain@gmail.com

\section{Francois Vasey}

CERN

E-mail: francois.vaseydcern.ch

In 2016, a TTC-PON (Timing, Trigger and Control system based on Passive Optical Networks) demonstrator was presented at TWEPP as an alternative to replace the TTC system, currently responsible for delivering timing, trigger and control commands in the LHC experiments. Towards a deployment foreseen for ALICE phase-1 upgrade, the system has been consolidated through flexible software implementation providing full configuration, complete calibration and extended monitoring and diagnostic tools. A new demonstrator setup was built with various FPGA platforms to test the system with an increased number of nodes and under different environmental conditions. This paper focuses on the TTC-PON system design with a discussion on its features and scaled-up tests.

Topical Workshop on Electronics for Particle Physics

11 - 14 September 2017

Santa Cruz, California

\footnotetext{
* Speaker.

†Corresponding author
} 


\section{Introduction}

The TTC-PON system was developed at CERN in the context of phase-1 and 2 upgrades of the LHC (large hadron collider) experiments in order to propose a new generation TTC system overcoming the major limitations of its predecessor [1]. The system is responsible for delivering timing, trigger and control from a central trigger unit to the detector sub-partitions in the back-end part of the LHC experiments as shown in figure 1. TTC-PON is based on passive optical networks technology, widely adopted in the telecommunications industry.

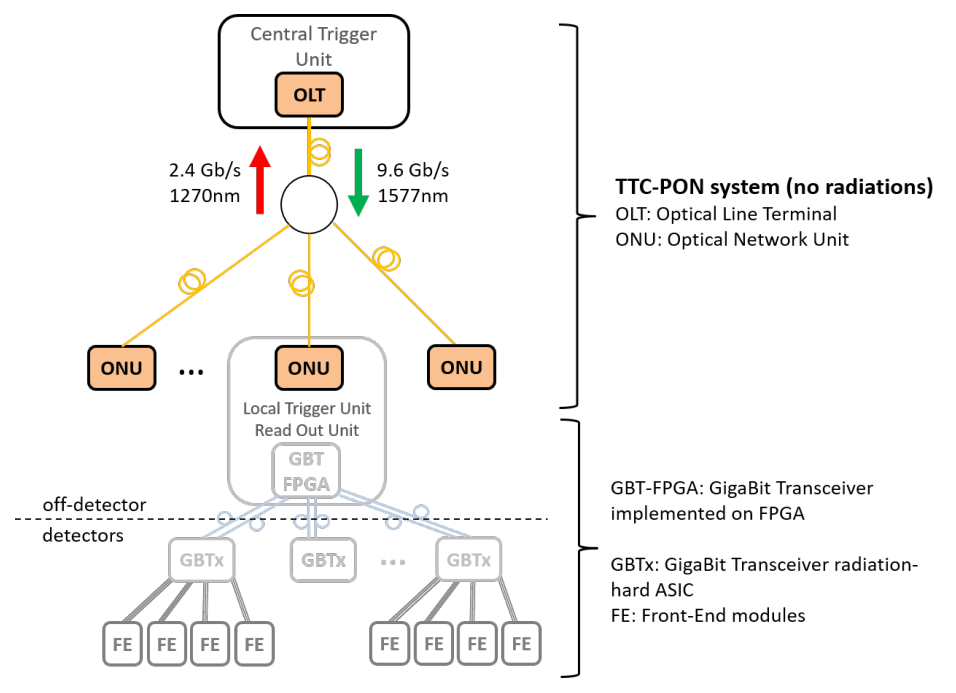

Figure 1: TTC-PON network in the context of an LHC experiment

TTC-PON is a point-to-multipoint bidirectional optical communication system in which a master node called OLT (optical line terminal) and slave nodes called ONU (optical network unit) can communicate through the same fiber using wavelength division multiplexing access. In the downstream direction, the OLT broadcasts information to the ONUs and in the upstream direction, the ONUs can send information to the OLT using a time division multiplexing scheme.

The TTC-PON concept has been studied and developed since 2010 [1] [2] and the current version of the system, based on the XG-PON (10-gigabit capable passive optical networks) technology from ITU (international tellecomunication union), will be adopted by the ALICE experiment for phase-1 upgrades [3].

\section{TTC-PON Protocol}

The principle behind TTC-PON is to make use of the industry mature optical components developed for the XG-PON standard and to build a custom protocol which targets CERN specific needs.

The downstream data transmission has a line rate of $9.6 \mathrm{~Gb} / \mathrm{s}$ and a wavelength at $1577 \mathrm{~nm}$. The framing scheme is constructed on a LHC bunch clock period basis ( $25 \mathrm{~ns})$ as shown in figure 2 top. Each frame is composed of four main fields namely: 8 bits of header (HDR) are used for frame 
alignment; 4 bits are for internal system control and monitoring (SC), which are assembled in a 36 bits frame and further protected by an error-detecting code; 200 bits are available to the final user and 28 bits are redundancy bits of a forward error correction (FEC) scheme. A self-synchronous scrambling scheme is adopted to provide DC-balance.

The upstream data transmission scheme is fully synchronous to the downstream transmission and has a line rate of $2.4 \mathrm{~Gb} / \mathrm{s}$ and a wavelength at $1270 \mathrm{~nm}$. It is based on a time division multiplexing scheme consisting of $125 \mathrm{~ns}$ time slots per ONU which are arbitrated in a round-robin manner with time offsets given by the OLT. The upstream framing is depicted in figure 2 bottom. Each burst is divided into five main fields: 140 bits of preamble are used for receiver settling time adjustment; 8 bits of header are used for frame alignment; 8 bits of address are for ONU identification (ADDR); 8 bits are used for slow control responses and 56 bits are at the end user disposal. An $8 \mathrm{~b} 10 \mathrm{~b}$ encoding scheme is used for the data bits providing DC-balance and error detection capability.

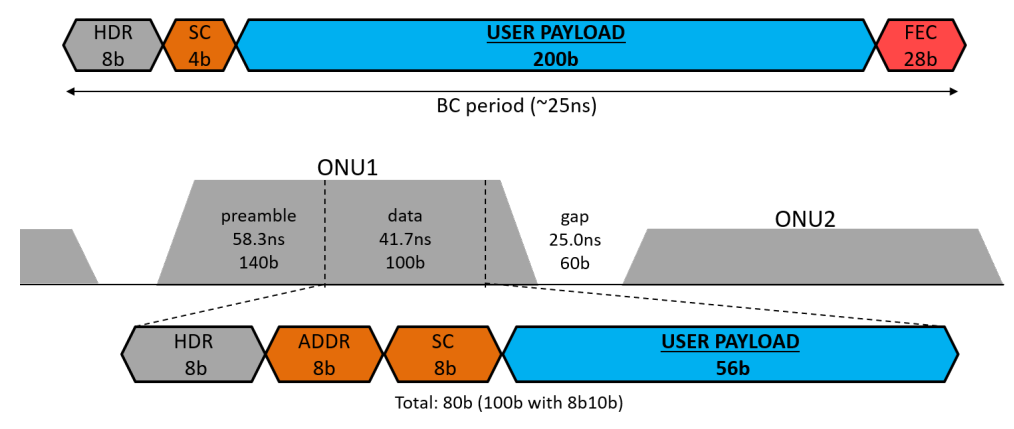

Figure 2: PON protocol: top - downstream framing, bottom - upstream framing

\section{System Implementation}

The system implementation is done in such a way that the system can be fully controlled and monitored from the OLT. Two FPGA soft IP cores (OLT and ONU) were developed for Kintex-7, Kintex Ultrascale and Arria10 devices. They are delivered with an example design which focuses on system characterization with BER (bit-error-ratio) tests and latency measurement capabilities. It also contains an example of how a TTC-PON user is supposed to interface the cores with his/her own system as shown in figure 3 .

The FPGA cores implement the high-speed encoding/decoding functions. System management (initialization and full monitoring) is implemented in software. It is important to note the portability of the software implementation which can be potentially migrated to a soft-processor or hard-processor in case of particular user needs.

In terms of specific hardware for TTC-PON system implementation, PON devices (optical transceivers in SFP+ package and passive optical splitters) are needed and an external PLL is recommended for the ONU. This PLL shall have a fixed input-to-output delay after reset and power cycles and is used for cleaning the downstream recovered clock to be used for upstream transmission and data forwarding. 


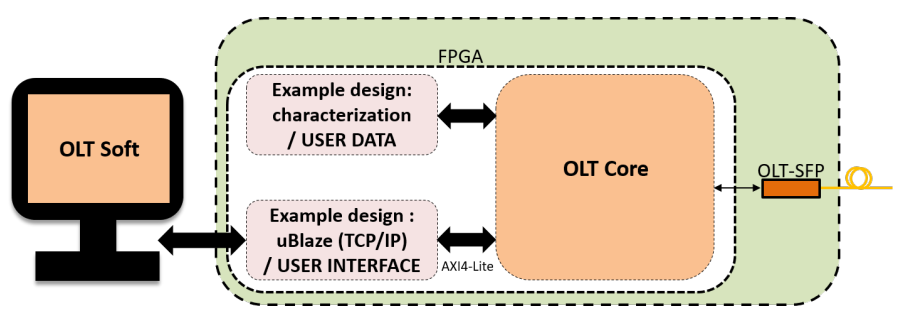

Figure 3: OLT IP core example

\section{System Operation}

When an ONU is connected to a TTC-PON network, it automatically locks to the incoming downstream data and the user starts receiving data and a stable clock. In the upstream path, it does not transmit before a software-based ranging procedure is performed by the OLT and a time slot is allocated for each ONU.

During a system run, a full set of monitoring tools are available allowing full diagnostic of the network. The available monitoring tools and their typical applications are summarized in table 1 .

\begin{tabular}{|c|c|c|}
\hline Monitoring type & Parameters & Typical applications \\
\hline Error monitor & $\begin{array}{l}\text { downstream error correction/detection activity } \\
\text { downstream eye scan } \\
\text { upstream error detection activity }\end{array}$ & link health analysis \\
\hline SFP monitor & $\begin{array}{l}\mathrm{Rx} / \mathrm{Tx} \text { power } \\
\text { tx bias, temperature }\end{array}$ & $\begin{array}{l}\text { preventive maintenance } \\
\text { degradation analysis }\end{array}$ \\
\hline Sticky Bits Interrupt & $\begin{array}{l}\text { MGT lock parameters } \\
\text { loss of lock down link } \\
\text { missing ONU burst }\end{array}$ & $\begin{array}{l}\text { debugging } \\
\text { analysis of system issues }\end{array}$ \\
\hline ONU burst phase & roundtrip variation (104ps bin size) & $\begin{array}{l}\text { burst drift analysis } \\
\text { recovered clock drift analysis }\end{array}$ \\
\hline
\end{tabular}

Table 1: system monitoring capabilities

\section{System Robustness}

The TTC-PON system has been extensively tested on a large demonstrator setup composed of one OLT implemented in a KCU105 evaluation board and eight ONUs implemented in KC705, KCU105 and Altera A10 GX evaluation boards as shown in figure 4. Long-duration BER tests were performed and also the impact of different conditions (temperature, dynamic range) were characterized.

An example of long BER vs OMA (optical modulation amplitude) test results is shown in figure 5. This test targets $B E R=10^{-13}$ in the downstream direction and $B E R=10^{-11}$ in the upstream direction and it was performed under ambient temperature. The ONU modules used in this test are from the same manufacturer. In the graph, 28 curves are displayed per ONU and the total test duration was 18 days. It is hard to observe the different curves for a given ONU as there is a good repeatability and consistency for all the ONUs. 


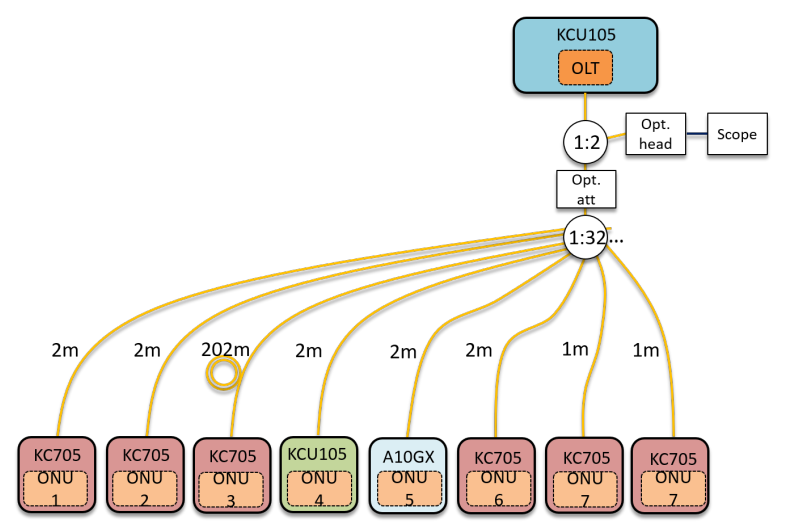

Figure 4: block diagram of extensive characterization setup demonstrating 1x64 split ratio

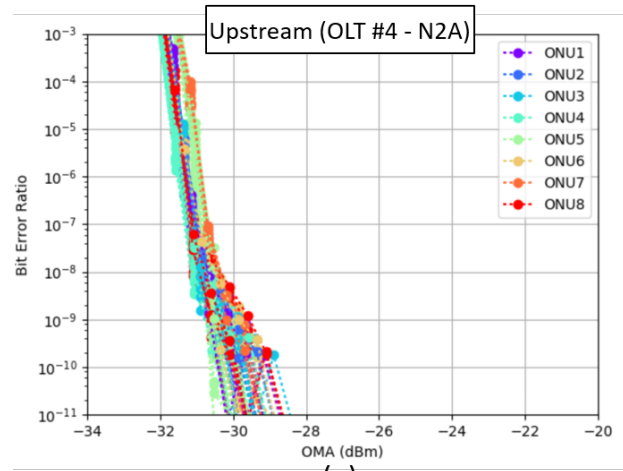

(a)

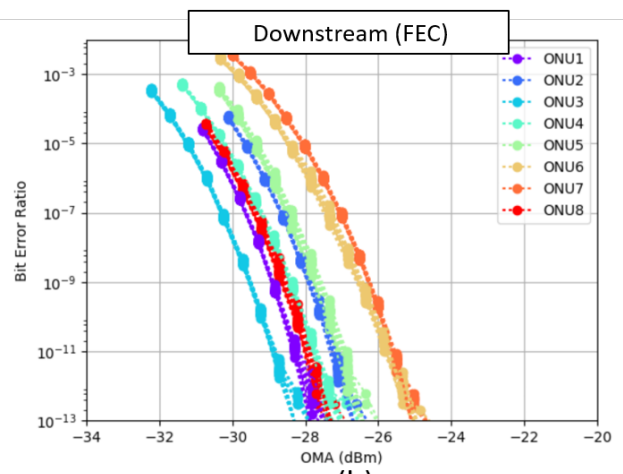

(b)

Figure 5: example long BER test results: (a) upstream (b) downstream

\section{Conclusions}

The TTC-PON system has achieved a mature development level and ALICE users have started to integrate it in their system. A price enquiry process has started and 100 OLT and $1000 \mathrm{ONU}$ modules will be purchased for upcoming network comissioning. The system is very flexible and possesses a complete set of monitoring tools. The system operation has been verified with an extended setup involving various FPGA platforms, optical modules from two different manufacturers and for different conditions.

\section{References}

[1] I. Papakonstantinou, C. Soos, S. Papadopoulos, S. Detraz, C. Sigaud, P. Stejskal et al., A fully bidirectional optical network with latency monitoring capability for the distribution of timing-trigger and control signals in high-energy physics experiments, IEEE Trans. Nucl. Sci. 58 (2011) 1628-1640.

[2] E. Mendes, S. Baron, D. Kolotouros, C. Soos and F. Vasey, The 10g ttc-pon: challenges, solutions and performance, Journal of Instrumentation 12 (2017) C02041.

[3] M. Krivda, D. Evans, K. Graham, A. Jusko, R. Lietava, O. Baillie et al., The alice trigger system for lhc run 3, 2018, https://indico.cern.ch/event/608587/contributions/2614820. 